\title{
TWITTER E INFORMACIÓN POLITICA EN LA PRENSA DIGITAL: LA RED SOCIAL COMO FUENTE DE DECLARACIONES EN LA ERA TRUMP
}

\author{
Twitter and political information in the main digital \\ media: The social network as a source of statements \\ in the Trump era
}

\section{Santiago Justel-Vázquez, Ariadna Fernández-Planells, María Victoria-Mas e Iván Lacasa-Mas}
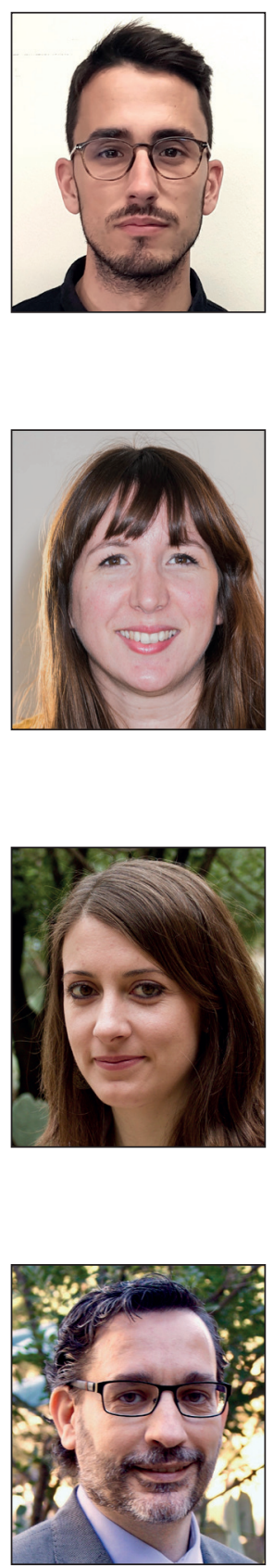

Santiago Justel-Vázquez, profesor en la Universitat Internacional de Catalunya, se doctoró en Comunicación por la Universitat Ramon Llull con una tesis centrada en la configuración de la agenda de los medios digitales y el interés público. Forma parte del grupo de investigación STReaM (Blanquerna-URL) en el que aborda temas relacionados con el periodismo digital, las redes sociales, la analítica web y las implicaciones de los cambios de los medios de comunicación en la esfera pública. Forma parte del proyecto Influencers en la comunicación política en España (CSO201788620-P), financiado por el Ministerio de Economía y Competitividad.

https://orcid.org/0000-0001-8068-6222

Universitat Internacional de Catalunya Inmaculada, 22. 08017 Barcelona, España sjustel@uic.es

Ariadna Fernández-Planells, doctora en Comunicación Pública por la Universitat Pompeu Fabra, centra su investigación en el estudio de la comunicación digital, la juventud y los movimientos sociales. Es profesora de la Universitat Politècnica de València (UPV) donde imparte asignaturas del Grado en Comunicación Audiovisual y del Master Universitario en Contenidos y Aspectos Legales en la Sociedad de la Información (Calsi). Forma parte del grupo de investigación Trademetrics, de la UPV, y del grupo JovisCom, de la UPF. Ha sido investigadora visitante en varias universidades de Perú y en la Cardiff School of Journalism, Media \& Cultural Studies en Gales.

https://orcid.org/0000-0003-4600-9345

Universitat Politècnica de València Paraninf, 1. 46730 Grau de Gandia (València), España arferpla@upv.es

María Victoria-Mas es profesora ayudante doctora en la Facultad de Ciencias de la Comunicación de la Universitat Internacional de Catalunya y visiting scholar en el Center for Media Engagement de la University of Texas (Austin). Es miembro del grupo de investigación 2017 SGR-1680 Comunicació, Mitjans i Societat (CMiS) y del proyecto Usos y preferencias informativas en el nuevo mapa de medios de España: audiencias, empresas, contenidos y gestión de la reputación en un entorno multipantalla, financiado por el Ministerio de Economía y Competitividad (CSO2015-64662-C4-1-R).

https://orcid.org/0000-0001-5107-0226

Universitat Internacional de Catalunya Inmaculada, 22. 08017 Barcelona, España mvictoria@uic.es

Iván Lacasa-Mas es doctor por la Universitat Autònoma de Barcelona (UAB) (Departament de Periodisme i Ciències de la Comunicació), licenciado en Filosofía por la Universitat de Barcelona (UB) y master en Estadística aplicada por la Universidad Rey Juan Carlos (URJC). Profesor titular de Teoría de la Comunicación en la Universitat Internacional de Catalunya (UIC) y visiting scholar en la University of Texas de Austin, donde es miembro del Center for Media Engagement y del Digital Media Research Project. Investigador principal del grupo Comunicació, Mitjans i Societat (Agaur, Gencat). Miembro del proyecto de investigación Usos y preferencias informativas en el nuevo mapa de medios en España (Ministerio de Economía y Competitividad).

https://orcid.org/0000-0002-9600-6049

Universitat Internacional de Catalunya Inmaculada, 22. 08017 Barcelona, España lacasa@uic.es 


\title{
Resumen
}

A través del análisis de contenido de una muestra de artículos de The New York Times publicados durante el primer año de legislatura del presidente de EUA, Donald Trump, la presente investigación cuantifica el número de veces que declaraciones realizadas a través de Twitter son utilizadas como fuente por los periodistas. Los resultados apuntan a que la red social se ha legitimado ya como fuente periodística en la prensa digital de referencia y de la información política, más allá de contextos electorales. El estudio contribuye a profundizar en los cambios que la red social ha introducido en el uso de fuentes periodísticas en el ámbito de las hard news.

\section{Palabras clave}

Periodismo; Información política; Comunicación política; Agenda; Medios de comunicación; Prensa digital; Fuentes de información; Redes sociales; Donald Trump; Twitter; The New York Times.

\begin{abstract}
Based on a sample of articles published by The New York Times in 2017 covering three important political issues, this research quantifies the number of tweets that end being used as journalistic sources. With the president of the United States, Donald Trump, as the main protagonist, the results point to the fact that Twitter statements are consolidated as a content used on a recurring basis also in the most reliable media. In this way, the study contributes to deepen into the changes that the social network has introduced in the use of journalistic sources in the field of hard news.
\end{abstract}

\section{Keywords}

Journalism; Political information; Political communication; Media agenda; Digital media; Information sources; Digital press; Social networks; Donald Trump; Twitter; The New York Times.

Justel-Vázquez, Santiago; Fernández-Planells, Ariadna; Victoria-Mas, María; Lacasa-Mas, Iván (2018). "Twitter e información política en la prensa digital: la red social como fuente de declaraciones en la era Trump". El profesional de la información, v. 27, n. 5, pp. 984-992.

https://doi.org/10.3145/epi.2018.sep.03

\section{Introducción}

El 11 de abril de 2018 la prensa internacional informaba de la intención de Estados Unidos de atacar Siria de manera inminente en respuesta al presunto uso de armas químicas contra la población civil por parte del régimen de Bashar al Assad. El origen de dicha información fue un mensaje en Twitter del presidente Donald Trump, quien consideró esa red social como el mejor canal para comunicarlo.

El uso creciente de Twitter que durante la última década han hecho los políticos se ha ido profesionalizando en un intento de influir más en la agenda mediática (Shapiro; Hempfil, 2017; Enli, 2017). A pesar de parecer espontánea e incluso poco profesional, la actividad que el nuevo presidente de EUA tuvo en esta plataforma ya durante su campaña electoral llevó a que los medios tradicionales citaran y discutieran sus tweets con asiduidad (Enli, 2017). Eso podría confirmar la legitimidad que, como otros canales de comunicación política más convencionales, ha adquirido Twitter como fuente periodística (Shapiro; Hempfil, 2017).

Estudios recientes apuntan a Twitter como uno de los canales habituales que los periodistas utilizan para monitorizar sus fuentes (Heravi; Harrower, 2016; English, 2016; Engesser; Humprecht, 2015; Pérez-Soler; Micó, 2015). Sin embargo, varios de esos trabajos concluyen que la utilización de tweets como declaraciones en la elaboración de noticias es algo más habitual en la prensa sensacionalista o en las secciones periodísticas llamadas "blandas" (soft) que en la prensa de calidad o en las noticias llamadas "duras" (hard) (Broersma; Graham, 2013; Paulussen; Harder, 2014).
A diferencia de lo que han hecho trabajos anteriores, esta investigación se centra en el uso que la prensa digital de calidad hace de Twitter. Nuestro objetivo principal será contrastar la hipótesis de que hoy esta red social ha pasado a ser una fuente de información política legitimada en las consideradas hard news.

Para contrastar esta hipótesis se ha realizado un análisis de contenido sobre una muestra de 233 artículos publicados por The New York Times (NYT) a lo largo de 2017 centrados en tres temas de actualidad de la política estadounidense:

- conflicto con Corea del Norte;

- cambio de rumbo de la administración Trump en materia de cambio climático;

- proyecto de construcción de un muro en la frontera entre EUA y México.

El primer año de legislatura de Donald Trump es un contexto temporal relevante, por la actividad continua del presidente americano en Twitter. Nos proponemos analizar si durante ese período uno de los referentes periodísticos internacionales como es el NYT ha utilizado un número significativo de tweets como fuente de información y cómo lo ha hecho (incrustados, citados...). Si se confirma que lo ha hecho, podremos afirmar que Twitter se ha consolidado o está en vías de consolidarse como uno más de los canales de comunicación política.

\section{Uso de Twitter por parte de los políticos}

A partir de 2009, políticos de todo el mundo adoptaron los medios sociales para involucrar a sus electores (Chi; Yang, 
2010). Durante la era Obama se vivió lo que se ha denominado la primera "elección de los medios sociales" (Enli, 2017 , p. 2). La campaña electoral de dicho presidente mostró el potencial de las aplicaciones 2.0 para "movilizar partidarios, difundir una narrativa y recaudar dinero" (Quinlan et al., 2018, p. 1031).

La experiencia de la campaña de Barack Obama se exportaría posteriormente a batallas electorales de países como Noruega o Reino Unido (Karlsen, 2013; Enli, 2017). Tal y como Hong y Nadler señalan,

"muchos se lanzaron a pronosticar que las redes sociales generarían una transformación radical en la relación entre políticos, gobiernos y ciudadanos" (Hong; Nadler, 2012, p. 456).

Sin embargo, la realidad retratada por la investigación académica ha resultado ser más modesta.

En el caso de Twitter, estudios realizados en Reino Unido y Holanda (Graham; Jackson; Broersma, 2016; Graham et al., 2013), Rumanía (Aparaschivei, 2011) o Estados Unidos (Enli; Naper, 2016), mostraron que a pesar de las posibilidades de interacción que ofrece, los políticos son reacios a establecer un diálogo con los votantes a través de este canal. Twitter se utiliza principalmente para la difusión de declaraciones, de información sobre las campañas y de propuestas políticas. Jackson y Lilleker afirmaron que en el Reino Unido los políticos siguen utilizando Twitter esencialmente para decir "mira lo mucho que trabajo" (Jackson; Lilleker 2011, p. 100). En España el papel predominante atribuido a esta red social por todos los políticos ha sido

"la difusión de las propuestas de sus programas electorales" (López-Meri; Marcos-García; Casero-Ripollés, 2017; p. 801), como si fuese "un medio de flujo unidireccional” (Aragón et al., 2013, p. 203).

Es decir, en varios países esta red social ha servido principalmente para amplificar formas de comunicación política más tradicionales como por ejemplo los comunicados o las ruedas de prensa.

La adopción de Twitter en comunicación política ha servido para amplificar formas de comunicación tradicionales, como los comunicados y las ruedas de prensa

La consolidación de Twitter como medio de comunicación política se ha producido a la par que la tendencia a la profesionalización o especialización de la gestión global de ese tipo de comunicación (Kreiss; Jasinski, 2012). Sin embargo, esta profesionalización de su uso no se ha dado de una manera homogénea (Quinlan et al., 2018). Por ejemplo en las últimas elecciones presidenciales de EUA, Enli (2017, p. 9) detectó que mientras la actividad en redes sociales de la campaña de Clinton

"confirmó las teorías sobre la profesionalización de las campañas electorales", la campaña de Trump "tuvo un estilo más amateur y auténtico".
Pew Research Center (2016) constató que Clinton y Sanders publicaron con la misma frecuencia que Trump pero que sus tweets y publicaciones, con un enfoque distinto, recibieron menor atención del público que los de Trump. Las controvertidas declaraciones del ahora presidente tuvieron además un impacto directo en los medios tradicionales que a menudo citaban y debatían sus tweets (Enli, 2017). El estatus de celebridad de Trump y su conocimiento de las lógicas de los medios de comunicación impulsaron su campaña y le permitieron

"una estrategia basada en un uso controvertido e inesperado" de Twitter (Enli, 2017, p. 11).

Por tanto a la hora de atraer la cobertura de los medios tradicionales no sólo influyó el uso que hicieron de la red social los candidatos demócratas, más similar al de otros medios de comunicación política convencionales. Como apunta Enli (2017, p. 18), también fue determinante "el capital" de Trump como candidato y su uso aparentemente espontáneo y poco estratégico de la red social.

Las investigaciones sobre la adopción de Twitter por parte de los políticos indican que éstos han sumado esta plataforma al conjunto de medios utilizados en sus estrategias de comunicación. Unas veces lo han hecho de manera más tradicional, otras de manera más novedosa y complementaria al resto de medios de comunicación convencionales.

Puesto que gran parte de los estudios previos sobre el uso de Twitter en la comunicación de los políticos se centran en el estudio de los períodos electorales, lo que aún conviene aclarar es hasta qué punto se ha normalizado su uso en contextos más ordinarios, como los que tienen lugar en el día a día de una legislatura. Es evidente que el objetivo principal de ese uso ya no es sólo predisponer al electorado, sino también compartir información con los periodistas e influir de modo habitual en la agenda de los medios más relevantes (Shapiro; Hemphill, 2017). Sin embargo, discernir hasta qué punto los políticos logran ese objetivo requiere comprobar la hipotética legitimación de Twitter como fuente, también en la información política publicada por los medios periodísticos.

\section{Uso de Twitter en la profesión periodística}

Los periodistas utilizan Twitter principalmente para compartir sus trabajos, para seguir las cuentas de sus fuentes o de otros medios, y para obtener material e ideas que permitan elaborar nuevas historias (Heravi; Harrower, 2016; Engesser; Humprecht, 2015; Pérez-Soler; Micó, 2015).

El uso de Twitter se ve reflejado en piezas periodísticas basadas en las declaraciones que determinadas fuentes realizan en la red social o en etiquetas (hashtags) que recogen cuáles son los temas noticiosos del momento (English, 2016; Heravi; Harrower, 2016). Aunque el porcentaje de los contenidos publicados por los medios en los que se hace referencia explícita a la red social no sea mayoritario, muchas investigaciones han constatado que el monitoreo de redes sociales ya forma parte de las rutinas diarias en las redacciones (English, 2016; Paulussen; Harder, 2014; Broersma; Graham, 2013). O sea, se incorpora al trabajo periodístico un nuevo medio de acceso a las fuentes y de verificación de 
información, que es más inmediato y accesible (Heravi; Harrower, 2016; Lecheler; Kruikemeier, 2016). Sin embargo, las investigaciones aún disienten sobre el grado y el modo en que los cambios en las prácticas profesionales están influyendo en el tratamiento de las fuentes o en los contenidos periodísticos, ya que muestran diferencias entre países, tipos de medios y sectores periodísticos (Deprez; Van-Leuven, 2017; Lecheler; Kruikemeier, 2016; Paulussen; Harder, 2014).

Algunos estudios previos sugieren que el uso de las redes sociales, y en concreto de Twitter, como fuente de información se consolidó primero para las noticias soft. Paulussen y Harder (2014) constataron que cuando la prensa del norte de Bélgica cita estas plataformas lo hace mayoritariamente en dicho ámbito y para referirse a ciudadanos, celebridades o personalidades del mundo del deporte. English (2016) confirma esa idea en periodismo deportivo en el Reino Unido y explica que los periodistas empezaron a adoptar Twitter como uno de sus medios principales de monitoreo de fuentes cuando algunos deportistas empezaron a hacer declaraciones relevantes sobre sus carreras en esta plataforma. Tales declaraciones eran más inmediatas, atractivas y cercanas que las tradicionales notas o ruedas de prensa.

Ese uso diferencial de Twitter que hace cierto tipo de fuentes, es en parte la causa de que sea más habitual encontrar tweets citados explícitamente en las noticias soft (Paulussen; Harder, 2014). En la misma línea Broersma y Graham (2013) afirmaron que Twitter ha logrado la diversificación de las voces que aparecen en los medios, lo cual implica una tendencia a la personalización o humanización de la información, aunque esta sea más propia de los tabloides que de la prensa de calidad. Estos autores afirman que el uso de Twitter en la prensa es mucho mayor en Reino Unido que en Alemania, debido a que el modelo británico es eminentemente comercial, especialmente en el caso de los tabloides, y a que su sistema mediático es mucho más competitivo. Eso les lleva a publicar más historias sensacionalistas y "Twitter es un medio personal por excelencia" que les provee de ese tipo de contenidos (Broersma; Graham, 2013, p. 460).

La investigación que se ha realizado hasta el momento no aclara hasta qué punto Twitter se ha legitimado como fuente en la prensa de calidad y en las noticias hard. Los estudios que analizan el tipo de fuentes que los periodistas siguen en Twitter afirman que la mayoría de ellas son otros medios de comunicación o expertos alejados de las noticias soft como por ejemplo la salud o la política (Deprez; Van-Leuven, 2017; Heravi; Harrower, 2016). Sin embargo, las investigaciones que se han centrado en analizar el contenido publicado por los medios han constatado que las referencias explícitas que hacen a Twitter como fuente periodística son minoritarias (Elena, 2018; Paulussen; Harder, 2014; Broersma; Graham, 2013). Un estudio reciente sobre el uso de las redes sociales en el periodismo internacional concluye que éstas han tenido una presencia escasa en las principales cadenas de información global (BBC World News, Al Jazeera English y CNN) en la cobertura de las revueltas de Egipto en 2011, Ucrania en 2013, y Venezuela en 2017 (Elena, 2018). Elena afirma que eso se debe a que los periodistas suelen omitir la citación de las redes sociales puesto que son una fuente que consideran deslegitimada (Elena, 2018) o a que las grandes empresas informativas tienen periodistas sobre el terreno realizando la cobertura, por lo que no requieren de esos otros recursos (Elena; Tulloch, 2017). Por el contrario, Shapiro y Hemphill (2017, p. 17) afirman que periodistas de medios tan importantes como The New York Times consideran los anuncios hechos por miembros del Congreso estadounidense a través de esta red como "un sustituto válido del comunicado de prensa tradicional".

En definitiva, las investigaciones realizadas hasta el momento permiten afirmar que el uso de Twitter como canal de monitorización y de verificación es común entre los periodistas de todos los medios. Tal hecho permite apuntar que las nuevas rutinas profesionales que esa red social ha originado han comenzado a modificar el tipo de fuentes informativas que la prensa utiliza. Sin embargo, no existen suficientes evidencias empíricas sobre la legitimización de esta red social en los medios de referencia, ni en las noticias hard. Las investigaciones existentes han detectado que la frecuencia de citación de Twitter en los contenidos y medios genuinamente periodísticos es muy baja. A pesar de ello, la adopción de la red social que ha hecho Trump al convertirse en presidente de los EUA hace pensar que su primer año de legislatura es un contexto temporal relevante para tratar de constatar nuestra hipótesis. El impacto que en el público y en los medios ha tenido su estrategia comunicativa, de un estilo más similar al que tienen otras fuentes más ligadas a las noticias soft, nos hacen suponer que sus tweets pueden haberse incluido con más facilidad en las informaciones hard y en los medios de referencia. Eso favorecería la inclusión de Twitter entre las fuentes que los periodistas consideran legitimadas.

\section{Se analiza en qué medida Twitter se ha consolidado como un canal utilizado por los periodistas para acceder a sus fuen- tes en información política}

\section{Metodología}

Con el objetivo principal de contrastar la hipótesis de que Twitter es hoy una fuente de información legitimada en información política, considerada hard news, esta investigación plantea un análisis de contenido centrado en el $N Y T$, uno de los medios de referencia a nivel mundial. A diferencia de estudios previos, que comparaban la presencia de Twitter en las soft news y en las hard news (Paulussen; Harder, 2014; Broersma; Graham, 2013) o que, centrados en las hard news, han analizado la transferencia de temas políticos de Twitter a la agenda de los medios tradicionales (Shapiro; Hemphill, 2017), este trabajo busca específicamente identificar la citación explícita de Twitter como fuente informativa en las informaciones políticas.

Concretamente nuestro análisis se enmarca en el primer año de legislatura de Trump para evitar la excepcionalidad comunicativa que rodea a los períodos electorales. Consideramos al actual presidente de los EUA un caso de estudio paradigmático por el uso preferencial que hace de Twitter. 
Se han tenido en cuenta las particularidades que tiene un análisis de contenido cuando se aplica a medios online, recogidas por varios investigadores (Widholm, 2015; Karlsson; Sjøvaag, 2016). Para garantizar que el estudio pueda ser revisado o replicado, incluso en caso de que el material deje de estar disponible online o sea modificado, se guardaron capturas de pantalla de cada uno de los items analizados. Se salvó así uno de los puntos que pueden complicar la aplicación del método del análisis de contenido a las noticias online, derivado del carácter "fluido y efímero" de los textos en la Web, el cual "plantea nuevas demandas a los investigadores" respecto a la metodología clásica (Karlsson; Sjøvaag, 2016).

En esta investigación se han analizado las noticias, piezas de opinión y análisis publicados por el NYT a lo largo de 2017 sobre tres temas de actualidad de la política estadounidense con evidente repercusión internacional:

- conflicto con Corea del Norte por su programa nuclear;

- decisión de EUA de retirarse del Acuerdo de París contra el cambio climático;

- proyecto de construcción del muro en la frontera EUA-México.

La selección de noticias y artículos se ha realizado a partir de una búsqueda avanzada en Google, restringida al dominio web del medio estudiado y ejecutada a través de las siguientes palabras clave:

\section{site:nytimes.com "trump" "north korea" site:nytimes.com "trump" "mexico" "wall" site:nytimes.com "trump" "climate change"}

Para la búsqueda se utilizaron palabras clave relacionadas con los tres conflictos que se querían estudiar. A los términos "north korea", "mexico", "wall" y "climate change" se añadió la palabra clave "Trump", pues se entendió que cualquier artículo que abordara estas cuestiones de la política exterior estadounidense iba a hacer referencia al que siempre es uno de los actores principales: el presidente de Estados Unidos, en el momento de la investigación, Donald Trump. Diferentes ensayos demostraron que la palabra clave "Trump" era más efectiva que términos como "U.S." o "United States" para filtrar y recuperar las noticias de interés de cada uno de los temas. Se eligieron pues esos términos de búsqueda, tras comprobar que no sólo iba a dar como resultado noticias centradas en Trump, sino las principales piezas sobre cada uno de los temas.

De los resultados obtenidos mediante las búsquedas se descartaron aquellos artículos que no se podrían enmarcar en el ámbito de la información política. Se descartaron también contenidos duplicados y noticias de agencia. En total se analizaron 233 artículos.

En cada artículo se analizó si citaba o contenía tweets. Mientras que estudios como el publicado por Broersma y Graham (2013) contabilizan el número de noticias que incluyen tweets como fuente, esta investigación cuantifica ese número de manera relativa al total de artículos publicados sobre un tema concreto en información política. Esta elección permite ofrecer porcentajes y por tanto cuantificar la magnitud del fenómeno dentro de dicha especialización periodística.

Se registró también, en caso de haberlos, de quién eran los tweets, el número que incluía cada pieza y si éste/éstos eran utilizados como fuente principal en la que se basa la noticia/ artículo.

Por medio de la plataforma Google Trends se seleccionaron los períodos temporales pertenecientes a 2017 en los que los tres temas seleccionados registraron una mayor actividad en internet (figura 1). Como el conflicto diplomático EUA-Corea del Norte generó interés en más momentos del año, para este tema se analizaron períodos más amplios y, por tanto, la muestra es mayor.

\section{Casi 1 de cada 10 artículos publicados en la edición digital de The New York Times $(8,6 \%)$ del total analizado incluyen un tweet como fuente principal}

De los 233 artículos de la muestra analizada, 171 tratan sobre el conflicto diplomático entre EUA y Corea del Norte, 27 sobre la construcción del muro en la frontera entre EUA y México, y 35 sobre el cambio de política en materia de cambio climático.

\section{Resultados}

A continuación presentamos los principales resultados obtenidos (figura 2).

78 de los 233 artículos que forman la muestra usan tweets como fuente o referencia - unas veces de forma literal, otras en forma de paráfrasis-. Esto representa 1 de cada 3 artículos: un 33,5\%.

En el análisis desglosado de cada tema no se aprecian gran-

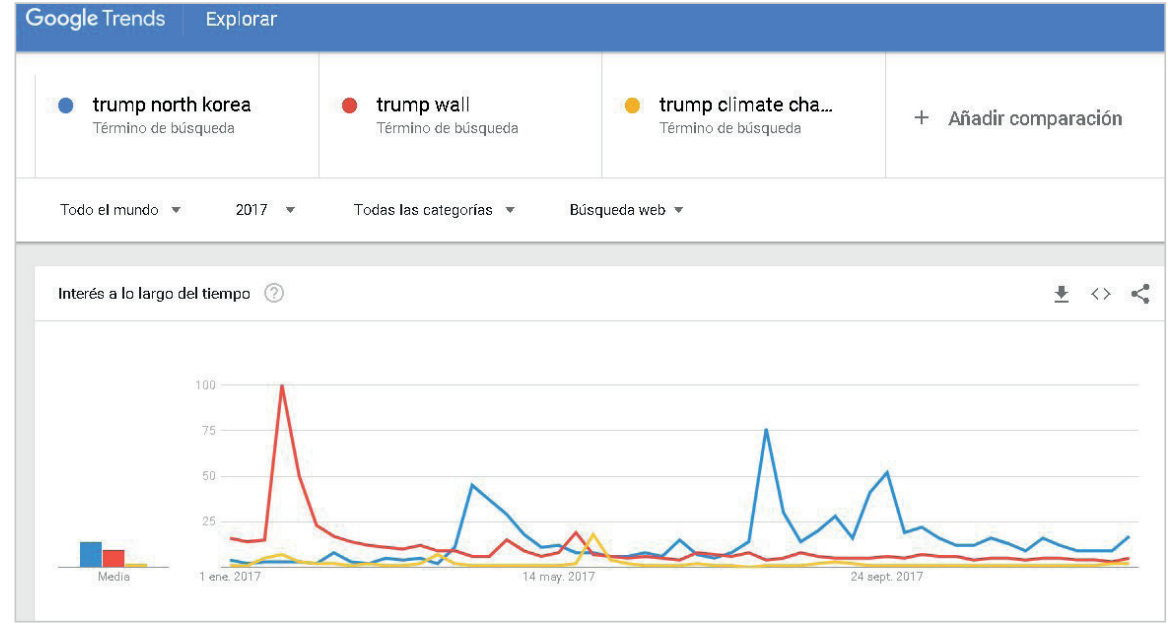

Figura 1. Intensidad de búsqueda de los temas de estudio. Fuente: Google Trends 
des diferencias en este porcentaje: un $32,7 \%, 37 \%$ y $34,1 \%$ de artículos utilizan declaraciones en Twitter sobre los temas de Corea del Norte, el muro en la frontera entre EUA y México, y el cambio climático, respectivamente.

En 1 de cada 4 artículos $(25,6 \%)$ de los que contienen tweets, éstos son la fuente principal. Si tomamos la muestra total de artículos (y no sólo aquellos que contienen tweets) nos encontramos con que casi 1 de cada 10 artículos (8,6\%) del total analizado usaron un tweet como fuente principal.

En un 3\% de todos los artículos se hace referencia específica en el titular a que la fuente principal del artículo es un tweet.

Entre los 78 artículos que usan material de Twitter como fuente de información, no todos referencian a un único tuit. El número de tweets referenciados varía: hay piezas que citan o incrustan hasta 8 .

47 artículos referencian 1 único tweet; 14 artículos contienen 2 tweets, 6 contienen 3 tweets, 5 contienen 4 tweets y 6 contienen 5 o más tweets.

En los artículos que sólo citan o incrus$\tan 1$ tweet, éste suele utilizarse como si fuese una declaración clásica, es decir, en sustitución o como complemento a una declaración de rueda de prensa, nota de prensa o declaración ante la prensa en un acto público o una entrevista. En muchos casos, estas declaraciones tienen un estilo espontáneo e incluso provocativo.

En 42 de los 47 artículos que sólo referencian un único tweet éste se cita y no se incrusta: la plataforma pierde protagonismo, podemos pensar que es porque lo importante no es el canal, sino lo que se ha dicho y quién lo ha dicho.

Cuando se referencian 3 o más tweets en una misma pieza, nos encontramos normalmente con noticias en las que se explica el diálogo mantenido en Twitter entre 2 políticos o en las que se muestra un recopilatorio de reacciones de personajes públicos ante un hecho. En este tipo de artículos los tweets son más protagonistas, son la base alrededor de la cual se construye la pieza.

Por lo que respecta al modo en que se incluye en los artículos la información publicada en Twitter, encontramos que un $30 \%$ de los tweets $(n=46)$ a los que se hace referencia se incrustan en los artículos. En el $70 \%$ restante $(n=107)$ únicamente se citan - directa o indirectamente- en el texto.

$\llbracket \frac{\pi}{\square \square}$

\section{Uso de tweets como fuente de información política}

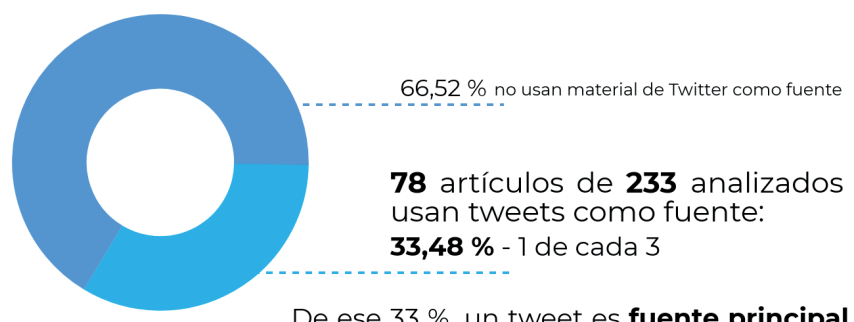
del artículo en 1 de cada 4 (25,6\%)

Respecto al total de la muestra, esto representa que casi 1 de cada 10 artículos (8,6\%) tienen como fuente principal un tweet

ब COREA DEL NORTE el 32, $7 \%$ de los artículos usan Twitter como fuente MURo méxico - EeUu $\quad$ el $\mathbf{3 7} \%$ de los artículos usan Twitter como fuente

CAMBIO CLIMÁTICO

el $\mathbf{3 4 , 1} \%$ de los artículos usan Twitter como fuente

Otros resultados destacados:

153 tweets repartidos en $\mathbf{7 8}$ artículos

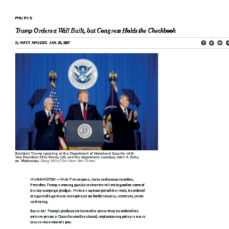

\begin{tabular}{|c|c|c|}
\hline 47 artículos & 口 & 1 tweet \\
\hline 14 artículos & $\square$ & 2 tweets \\
\hline 6 artículos & & 3 tweets \\
\hline 5 artículos & 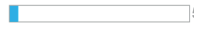 & 4 tweets \\
\hline 1 artículo & & 5 tweets \\
\hline 2 artículos & г & 6 tweets \\
\hline 1 artículo & $\square$ & 7 tweets \\
\hline artículos & & 8 tweets \\
\hline
\end{tabular}
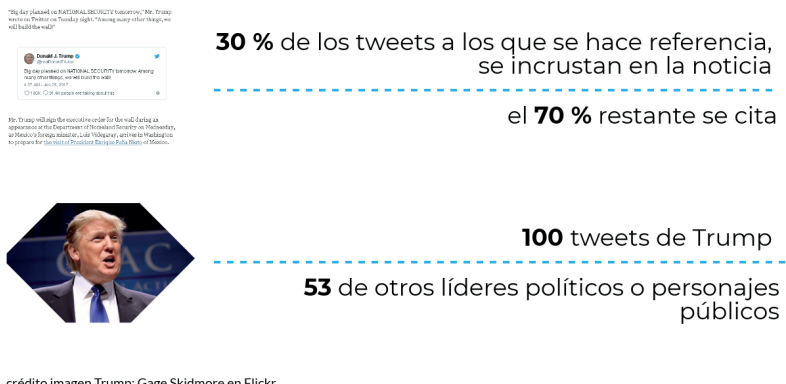

\section{PIKTOCHART}

Figura 2. Principales resultados 


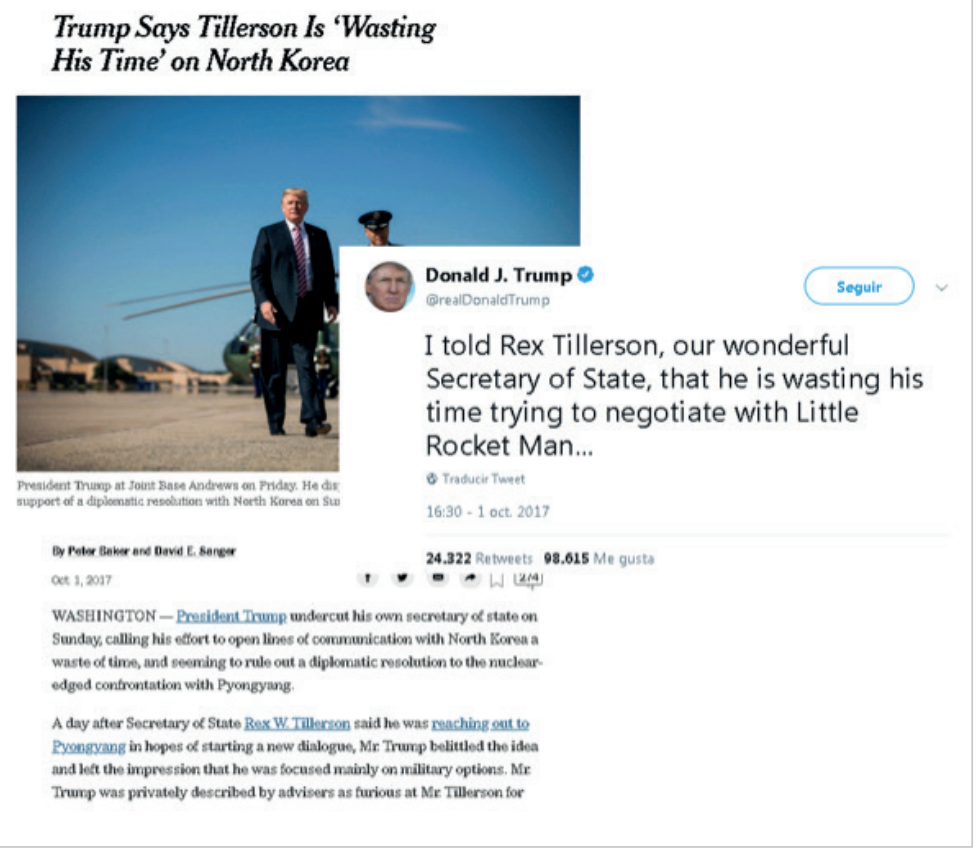

Figura 3. Noticia con un tweet como fuente principal

En un $65,3 \%$ de los casos en que se referencia material de Twitter, la fuente es un tweet de Donald Trump (un total de 100 tweets), mientras que en el $34,6 \%$ restante (53 tweets) se trata de otros líderes, personajes políticos, empresarios o celebridades. Esto se debe al tema de análisis escogido (política estadounidense), en el que el protagonista principal en tanto que máximo representante del gobierno americano es su presidente Donald Trump. Además hay que tener en consideración el uso intensivo que Trump hace de Twitter, tal y como hemos visto en el marco teórico.

\section{Discusión y conclusiones}

Investigaciones previas concluyeron que la utilización de tweets a modo de declaraciones en la elaboración de noticias era ya algo habitual en la prensa, pero especialmente en las soft news (English, 2016; Broersma; Graham, 2013).

En el caso de las hard news, estudios más recientes apuntan a que no se trata de una fuente ampliamente utilizada ni legitimada (Elena, 2018). Sin embargo la llegada de Donald Trump a la Casa Blanca y su particular estilo comunicativo a través de Twitter parecen haber contribuido, tal y como detectaron Shapiro y Hemphill (2017), a legitimar la red de microblogging como vehículo de comunicación política. Los anuncios y declaraciones de dicho presidente realizados en Twitter se han convertido en un complemento válido de otros canales de comunicación tradicional.

Nuestros resultados confirman la hipótesis de partida que apuntaba a la legitimación de Twitter como fuente periodística en medios de referencia y en secciones pertenecientes a las denominadas hard news. Concretamente ponen de relieve que las declaraciones publicadas en Twitter por políticos y personajes públicos son recogidas de forma recurrente por los periodistas de The New York Times como material para sus piezas de información política. La investigación no sólo constata la presencia de tweets en informaciones políticas de la prensa de calidad, sino que también muestra que no se trata de un fenómeno anecdótico. Twitter es fuente de declaraciones en 1 de cada 3 piezas analizadas y la fuente principal en casi 1 de cada 10. Al ejemplo citado en la introducción del artículo sobre el ataque a Siria, se añaden así muchos otros.

Los resultados también apuntan a que los tweets son utilizados por los periodistas como una declaración realizada a través de cualquier otra vía tradicional, sobre todo cuando se cita un único tweet en la información. En tal caso sustituyen o complementan a formas de comunicación política tradicionales como la comparecencia, la rueda de prensa o los comunicados (figura 4). Paralelamente han proliferado las piezas informativas que se nutren principalmente de tweets. Es el caso de aquellas que recogen el intercambio de declaraciones $u$ opiniones respecto a un tema político.

Es importante destacar también que el hecho de que un elevado número de tweets (30\%) sean incrustados directamente en las piezas informativas puede haber convertido a Twitter en un canal que promueve la cita a la fuente de manera más literal. De ser esto así, la fuente saldría reforzada en cuanto agenda-setter o creadora de la agenda, al conseguir introducir su mensaje en los medios sin ninguna edición por parte del periodista.

Para los periodistas, los tweets tienen la ventaja de que son fácilmente accesibles, más directos, atractivos y auténticos

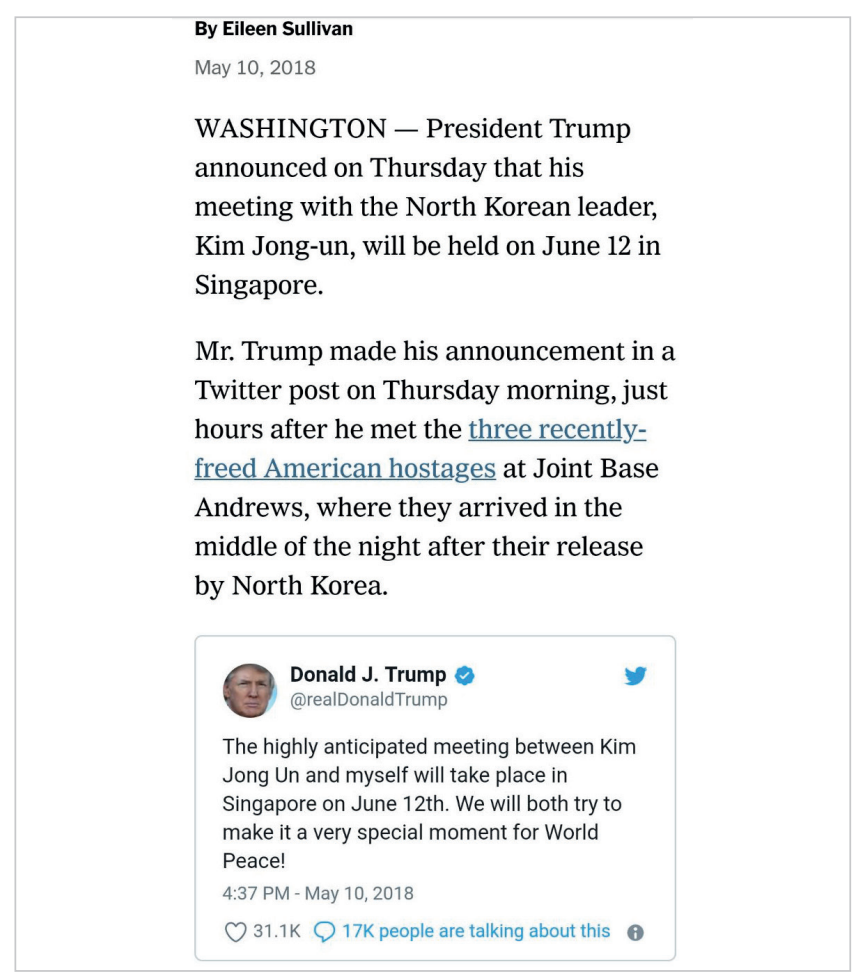

Figura 4. Fragmento de noticia del NYT que recoge el anuncio de Trump en Twitter sobre su intención de reunirse con el líder de Corea del Norte. 
(English, 2016; Paulussen; Harder, 2014) e incluso incorporables a la pieza incrustados como parte de la información. Nuestro estudio demuestra que esas ventajas ya no son aprovechadas sólo por los medios sensacionalistas y de modelo comercial (Broersma; Graham, 2013).

De todos modos conviene tener en cuenta el uso intensivo y aparentemente amateur o espontáneo que Trump hace de las redes sociales. Se asemeja así al de otro tipo de fuentes más relacionadas con las noticias soft (Enli, 2017). Por eso es más probable que sus mensajes en Twitter ya como presidente, hayan captado la atención del público y de los medios periodísticos. Pero hay que resaltar que esta estrategia no es exclusiva de Trump, sino que muchos otros políticos también usan Twitter como canal preferente para realizar determinados anuncios y posicionarse sobre acontecimientos utilizando un lenguaje más cercano e informal.

Los tweets se han convertido en una fuente periodística legitimada en medios de referencia y en las hard news

A pesar de la relevancia del contexto temporal y del caso escogido para nuestro trabajo, el estudio de sólo un caso no nos permite generalizar la hipótesis sobre la legitimación de Twitter como fuente en periodismo político en los medios de calidad. Puesto que tanto la adopción de los medios sociales por parte de los políticos (Quinlan et al., 2018), como por parte de los periodistas (Deprez; Van-Leuven, 2017; Lecheler; Kruikemeier, 2016) dista mucho de ser homogénea, convendrá que futuras investigaciones continúen el estudio sobre cómo, en otros contextos temporales y geográficos, unos y otros incorporan Twitter a sus rutinas profesionales. Además, dadas las peculiaridades del caso Trump, será necesario que tales futuras investigaciones analicen hasta qué punto se confirma la legitimación de esta red social en el caso de otros políticos y de sus distintas estrategias en dicha plataforma.

Aunque el uso intensivo que Donald Trump hace de Twitter puede llevarnos a pensar que al replicar el estudio en otro contexto político obtendríamos porcentajes inferiores, este hecho no resta relevancia a los resultados de nuestro estudio. Éstos constatan que el uso de la red social por parte de los políticos para hacer declaraciones públicas consigue hacerles entrar de un modo nuevo en la información publicada por los medios de referencia. La confirmación de que Twitter se ha convertido en una fuente legitimada para los periodistas, más allá de los períodos electorales, resulta relevante y demuestra la importancia de profundizar en las implicaciones que ello tiene para las rutinas periodísticas.

\section{Referencias}

Aparaschivei, Paul-Adrian (2011). "The use of new media in electoral campaigns: Analysis on the use of blogs, Facebook, Twitter and YouTube in the 2009 Romanian Presidential Campaign". Journal of media research, v. 4, n. 2, pp. 39-60. https://www.ceeol.com/content-files/document-134601.pdf
Aragón, Pablo; Kappler, Karolin-Eva; Kaltenbrunner, Andreas; Laniado, David; Volkovich, Yana (2013). "Communication dynamics in Twitter during political campaigns: The case of the 2011 Spanish national election". Policy \& internet, v. 5, n. 2, pp. 183-206.

http://airwiki.elet.polimi.it/images/7/70/Aragon_et_ al_2013_Twitter_Political_Campaigns.pdf https://doi.org/10.1002/1944-2866.POI327

Broersma, Marcel; Graham, Todd (2013). "Twitter as a news source: How Dutch and British newspapers used tweets in their news coverage, 2007-2011". Journalism practice, v. 7, n. 4, pp. 446-464.

https://doi.org/10.1080/17512786.2013.802481

Chi, Feng; Yang, Nathan (2010). Twitter in congress: Outreach vs transparency. University of Toronto.

https://mpra.ub.uni-muenchen.de/24060/1/MPRA_ paper_24060.pdf

Deprez, Annelore; Van-Leuven, Sarah (2017). "About pseudo quarrels and trustworthiness". Journalism studies, v. 19, n. 9, pp. 1257-1274. https://doi.org/10.1080/1461670X.2016.1266910

Elena, Martín (2018). "Los medios sociales como fuente de información en conflictos internacionales. Al-Jazeera English, BBC y CNN en Egipto 2011, Ucrania 2013 y Venezuela 2017". Trípodos, n. 43.

Elena, Martín; Tulloch, Christopher D. (2017). “Los social media como fuente de información en conflictos internacionales. Fuentes y praxis periodística en los informativos de la televisión de Cataluña durante la Primavera árabe egipcia". Hipertext.net, n. 15, pp. 46-56.

https://doi.org/10.2436/20.8050.01.45

Engesser, Sven; Humprecht, Edda (2015). "Frequency or skillfulness: How professional news media use Twitter in five Western countries". Journalism studies, v. 16, n. 4, pp. 513-529. https://doi.org/10.1080/1461670X.2014.939849

English, Peter (2016). "Twitter's diffusion in sports journalism: Role models, laggards and followers of the social media innovation". New media \& society, v. 18, n. 3, pp. 484-501. https://doi.org/10.1177/1461444814544886

Enli, Gunn (2017). "Twitter as arena for the authentic outsider: Exploring the social media campaigns of Trump and Clinton in the 2016 US presidential election". European journal of communication, v. 32, n. 1, pp. 50-61. https://doi.org/10.1177/0267323116682802

Enli, Gunn; Naper, Anja (2016). "Social media incumbent advantage: Barack Obama's and Mitt Romney's tweets in the 2012 U.S. Presidental election campaign". En: Bruns, Axel; Enli, Gunn; Skogerb $\varnothing$, Eli; Larsson, Anders-Olof; Christensen, Christian (eds.). The Routledge companion to social mdia and politics. New York: Routledge, pp. 364-377. ISBN: 9781138860766

Graham, Todd; Broersma, Marcel; Hazelhoff, Karin; Van'THaar, Guido (2013). "Between broadcasting political messages and interacting with voters: The use of Twitter during 
the 2010 UK general election campaign". Information, communication \& society, v. 16, n. 5, pp. 692-716.

https://core.ac.uk/download/pdf/96763067.pdf

https://doi.org/10.1080/1369118X.2013.785581

Graham, Todd; Jackson, Dan; Broersma, Marcel (2016).

“New platform, old habits? Candidates' use of Twitter during the 2010 British and Dutch general election campaigns". New media \& society, v. 18, n. 5, pp. 765-783.

http://eprints.whiterose.ac.uk/113492

https://doi.org/10.1177/1461444814546728

Heravi, Bahareh-Rahmanzadeh; Harrower, Natalie (2016). "Twitter journalism in Ireland: sourcing and trust in the age of social media". Information, communication \& society, v. 19, n. 9, pp. 1194-1213.

https://doi.org/10.1080/1369118X.2016.1187649

Hong, Sounman; Nadler, Daniel (2012). "Which candidates do the public discuss online in an election campaign?: The use of social media by 2012 presidential candidates and its impact on candidate salience". Government information quarterly, v. 29, n. 4, pp. 455-461.

https://doi.org/10.1016/j.giq.2012.06.004

Jackson, Nigel; Lilleker, Darren (2011). "Microblogging, constituency service and impression management: UK MPs and the use of Twitter". The journal of legislative studies, v. 17 , n. 1, pp. 86-105.

https://doi.org/10.1080/13572334.2011.545181

Karlsen, Rune (2013). “Obama's online success and European party organizations: Adoption and adaption of U.S. online practices in the Norwegian labor party". Journal of information technology and politics, v. 10, n. 2, pp. 158170.

https://doi.org/10.1080/19331681.2012.749822

Karlsson, Michael; Sjøvaag, Helle (2015) “Content analysis and online news: epistemologies of analysing the ephemeral Web". Digital journalism, v. 4, n. 1, pp. 177-192. https://doi.org/10.1080/21670811.2015.1096619

Kreiss, Daniel; Jasinski, Christopher (2016). "The tech industry meets presidential politics: Explaining the democratic party's technological advantage in electoral campaigning, 2004-2012". Political communication, v. 33, n. 4, pp. 1-19. https://doi.org/10.1080/10584609.2015.1121941
Lecheler, Sophie; Kruikemeier, Sanne (2016). "Re-evaluating journalistic routines in a digital age: A review of research on the use of online sources". New media \& society, v. 18, n. 1 , pp. 156-171. https://doi.org/10.1177/1461444815600412

López-Meri, Amparo; Marcos-García, Silvia; Casero-RipoIlés, Andreu (2017). "What do politicians do on Twitter? Functions and communication strategies in the Spanish electoral campaign of 2016". El profesional de la información, v. 26, n. 5, pp. 795-804.

https://doi.org/10.3145/epi.2017.sep.02

Paulussen, Steve; Harder, Raymond A. (2014). "Social media references in newspapers: Facebook, Twitter and YouTube as sources in newspaper journalism". Journalism practice, v. 8, n. 5, pp. 542-551.

https://goo.gl/jQyWSS

https://doi.org/10.1080/17512786.2014.894327

Pérez-Soler, Susana; Micó, Josep-Lluís (2015). “El mito de la conversación global. Usos de Twitter en las redacciones periodísticas catalanas y belgas". El profesional de la información, v. 24, n. 3, pp. 246-255.

https://doi.org/10.3145/epi.2015.may.04

Pew Research Center (2016). Election 2016: Campaigns as a direct source of news.

http://assets.pewresearch.org/wp-content/uploads/ sites/13/2016/07/PJ_2016.07.18_election-2016_FINAL.pdf

Quinlan, Stephen; Gummer, Tobias; Roßmann, Josh; Wolf, Christof (2018). "'Show me the money and the party!'-variation in Facebook and Twitter adoption by politicians". Information, communication \& society, v. 21, n. 8, pp. 10311049.

https://doi.org/10.1080/1369118X.2017.1301521

Shapiro, Matthew A.; Hemphill, Libby (2017). "Politicians and the policy agenda: Does use of Twitter by the US Congress direct New York Times content?". Policy \& internet, v. 9, n. 1, pp. 109-132.

https://doi.org/10.1002/poi3.120

Widholm, Andreas (2016). "Tracing online news in motion: Time and duration in the study of liquid journalism". Digital journalism, v. 4, n. 1, pp. 24-40.

https://doi.org/10.1080/21670811.2015.1096611

\section{Cronología de la Documentación Española}

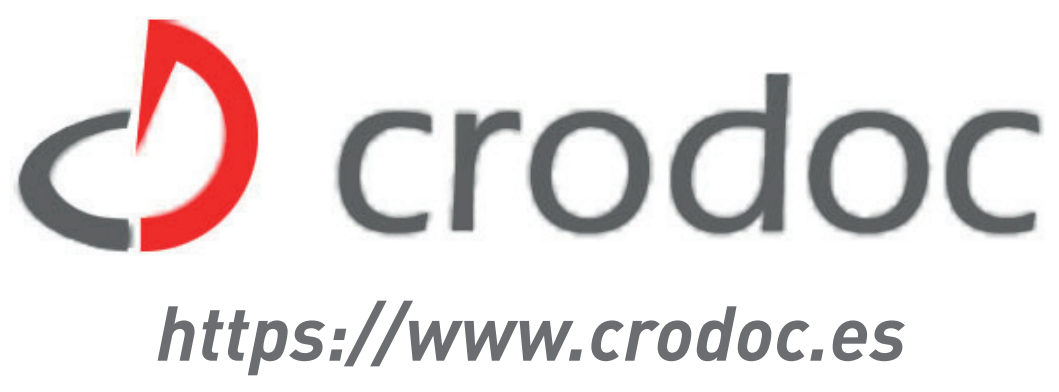




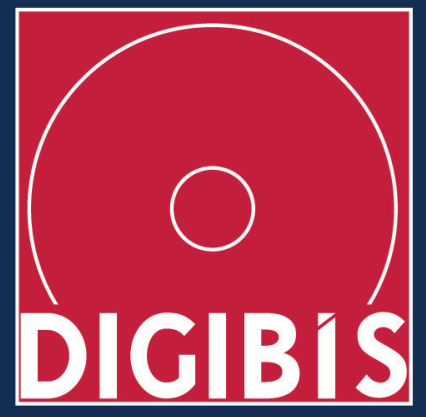

Nuestra vocación: contribuir a que el patrimonio cultural español sea preservado digitalmente y se difunda de la manera más amplia posible

Con metadatos ajustados a la normativa internacional

Aplicaciones Linked Open Data (LOD) con Reconciliación Semántica

Aplicaciones que pueden incorporar Recolector y Repositorio OAI-PMH

Aplicaciones pensadas y desarrolladas para hacer más eficiente y ágil el trabajo de los administradores y más comprensible para el usuario el acceso a los recursos catalogados. 